KYUNGPOOK Math. J. 49(2009), 521-532

\title{
On Hilbert-type Integral Inequalities with the Homogenous Kernel of -4 -degree
}

QILIANG HuAng* AND Bing HE

Department of Mathematics, Guangdong Institute of Education, Guangzhou, 510303

P. R. China

e-mail : qlhuang@yeah.net and hzs314@163.com

ABSTRACT. In this paper, by introducing a homogenous kernel of $-4-$ degree, we establish a new Hilbert-type integral inequality with multi-parameter and a best constant factor. As applications, the equivalent form, the reverse forms and some particular results are given correspondingly.

\section{Introduction}

In 1908, D. Hilbert established the following well known Hilbert's inequality (see $[1])$ : If $f(x), g(x) \geq 0$, such that $0<\int_{0}^{\infty} f^{2}(x) d x<\infty$ and $0<\int_{0}^{\infty} g^{2}(x) d x<\infty$, then

$$
\int_{0}^{\infty} \int_{0}^{\infty} \frac{f(x) g(y)}{x+y} d x d y<\pi\left\{\int_{0}^{\infty} f^{2}(x) d x \int_{0}^{\infty} g^{2}(x) d x\right\}^{\frac{1}{2}}
$$

where the constant factor $\pi$ is the best possible. Inequality (1.1) is important in analysis and its applications (see [2]). Under the same conditions of (1.1), we have (see $[1])$

$$
\begin{aligned}
& \int_{0}^{\infty} \int_{0}^{\infty} \frac{f(x) g(y)}{\max \{x, y\}} d x d y<4\left\{\int_{0}^{\infty} f^{2}(x) d x \int_{0}^{\infty} g^{2}(x) d x\right\}^{\frac{1}{2}} \\
& \int_{0}^{\infty} \int_{0}^{\infty} \frac{\ln (x / y)}{x-y} f(x) g(y) d x d y<\pi^{2}\left\{\int_{0}^{\infty} f^{2}(x) d x \int_{0}^{\infty} g^{2}(x) d x\right\}^{\frac{1}{2}}
\end{aligned}
$$

Inequality (1.2) and (1.3) are called Hilbert-type integral inequality. All the inequalities above are with the homogeneous kernel of -1 -degree. In 1998, Yang (see [3]-[4]) introduced a parameter $\lambda>0$ and the Beta function $B(u, v)$, and established the generalized form of (1.1) with the best constant factor $B\left(\frac{\lambda}{2}, \frac{\lambda}{2}\right)$ as

$$
\int_{0}^{\infty} \int_{0}^{\infty} \frac{f(x) g(y)}{(x+y)^{\lambda}} d x d y<B\left(\frac{\lambda}{2}, \frac{\lambda}{2}\right)\left\{\int_{0}^{\infty} x^{1-\lambda} f^{2}(x) d x \int_{0}^{\infty} x^{1-\lambda} g^{2}(x) d x\right\}^{1 / 2}
$$

* Corresponding author.

Received April 15, 2008 ; accepted July 9, 2008.

2000 Mathematics Subject Classification: 26D15.

Key words and phrases: Hilbert-type integral inequality, kernel, weight function, equivalent form, reverse form. 
Inequality(1.4) becomes into the following inequality when $\lambda=4$

$$
\int_{0}^{\infty} \int_{0}^{\infty} \frac{f(x) g(y)}{(x+y)^{4}} d x d y<\frac{1}{6}\left\{\int_{0}^{\infty} \frac{1}{x^{3}} f^{2}(x) d x \int_{0}^{\infty} \frac{1}{x^{3}} g^{2}(x) d x\right\}^{1 / 2} .
$$

A lot of generalized of the Hilbert-type inequalities appeared in the literature (see [5]-[11]) with parameters base on all the above inequalities. In this article, by introducing the parameters $a, b, c \in R_{+}$, we establish a new Hilbert-type integral inequality with the homogeneous kernel of -4 -degree and the best constant factor. At the same time, the inequality is generalized by dealing with a parameter $\lambda$. As applications, the equivalent form, the reverse forms and some particular results are considered correspondingly.

\section{Some lemmas}

Lemma 2.1. If $\widetilde{A}, \widetilde{B}, \widetilde{C} \in R, a, b, c \in R_{+}$and $\widetilde{A}+\widetilde{B}+\widetilde{C}=0$, then

$$
\lim _{x \rightarrow \infty}[\widetilde{A} \ln (x+a)+\widetilde{B} \ln (x+b)+\widetilde{C} \ln (x+c)]=0 .
$$

Proof. Since $\widetilde{C}=-\widetilde{A}-\widetilde{B}$, we get

$$
\begin{aligned}
& \lim _{x \rightarrow \infty}[\widetilde{A} \ln (x+a)+\widetilde{B} \ln (x+b)+\widetilde{C} \ln (x+c)] \\
&=\lim _{x \rightarrow \infty}\left[\widetilde{A} \ln \left(\frac{x+a}{x+c}\right)+\widetilde{B} \ln \left(\frac{x+b}{x+c}\right)\right]=0 .
\end{aligned}
$$

Lemma 2.2. Note $R_{+}^{4}=(0, \infty) \times(0, \infty) \times(0, \infty) \times(0, \infty)$, setting the parameter $\theta=(\lambda, a, b, c) \in R_{+}^{4}, a, b, c$ is not equal each other and $(x, y) \in(0, \infty) \times(0, \infty)$. Define the weight functions as

$$
\begin{aligned}
& \omega_{1}(x, \theta):=\int_{0}^{\infty} \frac{x^{2 \lambda} y^{2 \lambda-1}}{\left(x^{\lambda}+a y^{\lambda}\right)\left(x^{\lambda}+b y^{\lambda}\right)\left(x^{\lambda}+c y^{\lambda}\right)^{2}} d y \\
& \omega_{2}(y, \theta):=\int_{0}^{\infty} \frac{x^{2 \lambda-1} y^{2 \lambda}}{\left(x^{\lambda}+a y^{\lambda}\right)\left(x^{\lambda}+b y^{\lambda}\right)\left(x^{\lambda}+c y^{\lambda}\right)^{2}} d x .
\end{aligned}
$$

Then the above two integrals are convergent. Moreover, we get

$$
\omega_{1}(x, \theta)=\omega_{2}(y, \theta)=K(\theta):=\frac{1}{\lambda(a-c)(c-b)}+\frac{1}{\lambda} \ln \left(\frac{c^{\widetilde{A}+\widetilde{B}}}{a^{\widetilde{A}} b^{\widetilde{B}}}\right),
$$

where $\widetilde{A}=\frac{a}{(a-b)(a-c)^{2}}, \widetilde{B}=\frac{b}{(b-a)(b-c)^{2}}$ and $K(\theta)>0$.

Proof. Setting $u=\left(\frac{x}{y}\right)^{\lambda}$, by simple calculating, the two integrals of (2.2) turn into

$$
\omega_{1}(x, \theta)=\omega_{2}(y, \theta)=\frac{1}{\lambda} \int_{0}^{\infty} \frac{u}{(u+a)(u+b)(u+c)^{2}} d u .
$$


Obviously, the above integral is independent of $x, y$. The integrand of (2.4) can be decomposed into several parts

$$
\frac{u}{(u+a)(u+b)(u+c)^{2}}=\frac{\widetilde{A}}{u+a}+\frac{\widetilde{B}}{u+b}+\frac{\widetilde{C}}{u+c}+\frac{\widetilde{D}}{(u+c)^{2}},
$$

and it follows

$\widetilde{A}(u+b)(u+c)^{2}+\widetilde{B}(u+a)(u+c)^{2}+\widetilde{C}(u+a)(u+b)(u+c)+\widetilde{D}(u+a)(u+b)=u$,

Letting $u=-a,-b,-c$ respectively, we obtain $\widetilde{A}=\frac{a}{(a-b)(a-c)^{2}}, \widetilde{B}=$ $\frac{b}{(b-a)(b-c)^{2}}, \widetilde{D}=\frac{c}{(a-c)(c-b)}$. Then setting $u=0$, we get $\widetilde{A} b c^{2}+\widetilde{B} a c^{2}+$ $\widetilde{C} a b c+\widetilde{D} a b=0$. After that, put $\widetilde{A}, \widetilde{B}, \widetilde{D}$ into the above equality, we have $\widetilde{A}+\widetilde{B}+\widetilde{C}=0$. In fact,

$$
\begin{aligned}
\widetilde{C} & =\frac{1}{(a-c)(b-c)}+\frac{c}{(a-b)(b-c)^{2}}-\frac{c}{(a-b)(a-c)^{2}} \\
& =\frac{1}{(a-c)(b-c)}+\frac{c(a-b)(a+b-2 c)}{(a-b)(b-c)^{2}(a-c)^{2}}=\frac{a b-c^{2}}{(b-c)^{2}(a-c)^{2}}, \\
\widetilde{A}+\widetilde{B} & =\frac{a(b-c)^{2}-b(a-c)^{2}}{(a-b)(b-c)^{2}(a-c)^{2}}=\frac{a\left(b^{2}+c^{2}\right)-b\left(a^{2}+c^{2}\right)}{(a-b)(b-c)^{2}(a-c)^{2}} \\
& =\frac{a b(b-a)+(a-b) c^{2}}{(a-b)(b-c)^{2}(a-c)^{2}}=-\widetilde{C} .
\end{aligned}
$$

By the results above and considering (2.1), we get

$$
\begin{aligned}
0 & <\int_{0}^{\infty} \frac{u}{(u+a)(u+b)(u+c)^{2}} d u \\
& =\left.\left(\widetilde{A} \ln (x+a)+\widetilde{B} \ln (x+b)+\widetilde{C} \ln (x+c)-\frac{\widetilde{D}}{u+c}\right)\right|_{0} ^{\infty} \\
& =\frac{\widetilde{D}}{c}-\widetilde{A} \ln a-\widetilde{B} \ln b+\widetilde{A} \ln c+\widetilde{B} \ln c \\
& =\frac{1}{(a-c)(c-b)}+\widetilde{A} \ln \left(\frac{c}{a}\right)+\widetilde{B} \ln \left(\frac{c}{b}\right)<\infty .
\end{aligned}
$$

Hence by $(2.4),(2.3)$ is correct, and $K(\theta)>0$.

Lemma 2.3. Setting $p \in R^{1}-\{0,1\}, \frac{1}{p}+\frac{1}{q}=1, \theta=(\lambda, a, b, c) \in R_{+}^{4}, 0<\varepsilon<\lambda|p|$, $a, b, c$ is not equal each other, $K(\theta)$ is taken as the definition of (2.3), then

$$
I:=\varepsilon \int_{1}^{\infty} \int_{1}^{\infty} \frac{x^{2 \lambda-1-\frac{\varepsilon}{p}} y^{2 \lambda-1-\frac{\varepsilon}{q}}}{\left(x^{\lambda}+a y^{\lambda}\right)\left(x^{\lambda}+b y^{\lambda}\right)\left(x^{\lambda}+c y^{\lambda}\right)^{2}} d x d y=K(\theta)+o(1)\left(\varepsilon \rightarrow 0^{+}\right) .
$$


Proof. Setting $u=\left(\frac{x}{y}\right)^{\lambda}$, then

$$
\begin{aligned}
I= & \varepsilon \int_{1}^{\infty}\left[\int_{1}^{\infty} \frac{x^{2 \lambda-1-\frac{\varepsilon}{p}} y^{2 \lambda-1-\frac{\varepsilon}{q}}}{\left(x^{\lambda}+a y^{\lambda}\right)\left(x^{\lambda}+b y^{\lambda}\right)\left(x^{\lambda}+c y^{\lambda}\right)^{2}} d x\right] d y \\
= & \varepsilon \int_{1}^{\infty} y^{-1-\varepsilon}\left[\frac{1}{\lambda} \int_{y^{-\lambda}}^{\infty} \frac{u^{1-\frac{\varepsilon}{\lambda p}}}{(u+a)(u+b)(u+c)^{2}} d u\right] d y \\
= & \varepsilon \int_{1}^{\infty} y^{-1-\varepsilon}\left[\frac{1}{\lambda} \int_{0}^{\infty} \frac{u^{1-\frac{\varepsilon}{\lambda p}}}{(u+a)(u+b)(u+c)^{2}} d u\right] d y \\
& -\varepsilon \int_{1}^{\infty} y^{-1-\varepsilon}\left[\frac{1}{\lambda} \int_{0}^{y^{-\lambda}} \frac{u^{1-\frac{\varepsilon}{\lambda p}}}{(u+a)(u+b)(u+c)^{2}} d u\right] d y .
\end{aligned}
$$

Since $1-\frac{\varepsilon}{\lambda p}>0$, then $\frac{u^{1-\frac{\varepsilon}{\lambda p}}}{(u+a)(u+b)(u+c)^{2}} \leq \frac{1}{a b c^{2}}(0 \leq u \leq 1)$ and $\frac{u^{1-\frac{\varepsilon}{\lambda p}}}{(u+a)(u+b)(u+c)^{2}}<\frac{1}{u^{2}}(u \geq 1)$. So $\int_{0}^{\infty} \frac{u^{1-\frac{\varepsilon}{\lambda p}}}{(u+a)(u+b)(u+c)^{2}} d u$ is uniform convergent in $\varepsilon \in(0, \lambda|p|)$. Since the integrand of the integral is continuous about $\varepsilon$, by $(2.3)$ and (2.4), we have

$$
\frac{1}{\lambda} \int_{0}^{\infty} \frac{u^{1-\frac{\varepsilon}{\lambda p}}}{(u+a)(u+b)(u+c)^{2}} d u=K(\theta)+o(1)\left(\varepsilon \rightarrow 0^{+}\right) .
$$

By (2.6) and (2.7), it follows

$$
\begin{aligned}
I & <\varepsilon \int_{1}^{\infty} y^{-1-\varepsilon}(K(\theta)+o(1)) d y=K(\theta)+o(1) \\
I & >\varepsilon \int_{1}^{\infty} y^{-1-\varepsilon}(K(\theta)+o(1)) d y-\varepsilon \int_{1}^{\infty} y^{-1}\left(\frac{1}{\lambda} \int_{0}^{y^{-\lambda}} \frac{1}{a b c^{2}} d u\right) d y \\
& =K(\theta)+o(1)-\frac{\varepsilon}{\lambda a b c^{2}} \int_{1}^{\infty} y^{-1-\lambda} d y=K(\theta)+o(1)-\frac{\varepsilon}{\lambda^{2} a b c^{2}}
\end{aligned}
$$

Letting $\varepsilon \rightarrow 0^{+}$and by (2.8), (2.9), we get $\lim _{\varepsilon \rightarrow 0^{+}} I=K(\theta)$, and (2.5) is correct.

\section{Main results and the equivalent forms}

Theorem 3.1. If $p>1, \frac{1}{p}+\frac{1}{q}=1, \theta=(\lambda, a, b, c) \in R_{+}^{4}, a, b, c$ is not equal each other, $K(\theta)$ is taken as the definition of (2.3), $f(x), g(x) \geq 0$ such that $0<$ $\int_{0}^{\infty} x^{p(1-2 \lambda)-1} f^{p}(x) d x<\infty$ and $0<\int_{0}^{\infty} y^{q(1-2 \lambda)-1} g^{q}(x) d x<\infty$, then

$$
\begin{aligned}
\int_{0}^{\infty} \int_{0}^{\infty} \frac{f(x) g(y)}{\left(x^{\lambda}+a y^{\lambda}\right)\left(x^{\lambda}+b y^{\lambda}\right)\left(x^{\lambda}+c y^{\lambda}\right)^{2}} d x d y \\
(3.1) \quad<K(\theta)\left\{\int_{0}^{\infty} x^{p(1-2 \lambda)-1} f^{p}(x) d x\right\}^{1 / p}\left\{\int_{0}^{\infty} x^{q(1-2 \lambda)-1} g^{q}(x) d x\right\}^{1 / q}
\end{aligned}
$$


where the constant factor $K(\theta)$ is the best possible. In particular, taking $\lambda=1$, we have

$$
\begin{aligned}
\int_{0}^{\infty} \int_{0}^{\infty} & \frac{f(x) g(y)}{(x+a y)(x+b y)(x+c y)^{2}} d x d y \\
& <K(1, a, b, c)\left\{\int_{0}^{\infty} \frac{1}{x^{p+1}} f^{p}(x) d x\right\}^{1 / p}\left\{\int_{0}^{\infty} \frac{1}{x^{q+1}} g^{q}(x) d x\right\}^{1 / q}
\end{aligned}
$$

Proof. By Hölder's inequality with weight (see [12]) and (2.2), (2.3), we have

$$
\begin{aligned}
\int_{0}^{\infty} & \int_{0}^{\infty} \frac{f(x) g(y)}{\left(x^{\lambda}+a y^{\lambda}\right)\left(x^{\lambda}+b y^{\lambda}\right)\left(x^{\lambda}+c y^{\lambda}\right)^{2}} d x d y \\
= & \int_{0}^{\infty} \int_{0}^{\infty} \frac{1}{\left(x^{\lambda}+a y^{\lambda}\right)\left(x^{\lambda}+b y^{\lambda}\right)\left(x^{\lambda}+c y^{\lambda}\right)^{2}}\left[\frac{x^{\frac{1-2 \lambda}{q}}}{y^{\frac{1-2 \lambda}{p}}} f(x)\right]\left[\frac{y^{\frac{1-2 \lambda}{p}}}{x^{\frac{1-2 \lambda}{q}}} g(y)\right] d x d y \\
\leq & \left\{\int_{0}^{\infty} \int_{0}^{\infty} \frac{x^{(1-2 \lambda)(p-1)} y^{2 \lambda-1}}{\left(x^{\lambda}+a y^{\lambda}\right)\left(x^{\lambda}+b y^{\lambda}\right)\left(x^{\lambda}+c y^{\lambda}\right)^{2}} f^{p}(x) d x d y\right\}^{\frac{1}{p}} \\
& \times\left\{\int_{0}^{\infty} \int_{0}^{\infty} \frac{y^{(1-2 \lambda)(q-1)} x^{2 \lambda-1}}{\left(x^{\lambda}+a y^{\lambda}\right)\left(x^{\lambda}+b y^{\lambda}\right)\left(x^{\lambda}+c y^{\lambda}\right)^{2}} g^{q}(y) d x d y\right\}^{\frac{1}{q}} \\
= & \left\{\int_{0}^{\infty} \omega_{1}(x, \theta) x^{p(1-2 \lambda)-1} f^{p}(x) d x\right\}^{1 / p}\left\{\int_{0}^{\infty} \omega_{2}(y, \theta) y^{q(1-2 \lambda)-1} g^{q}(y) d y\right\}^{1 / q} \\
(3.3)= & K(\theta)\left\{\int_{0}^{\infty} x^{p(1-2 \lambda)-1} f^{p}(x) d x\right\}^{1 / p}\left\{\int_{0}^{\infty} x^{q(1-2 \lambda)-1} g^{q}(x) d x\right\}^{1 / q} .
\end{aligned}
$$

If (3.3) takes the form of the equality, then there exist constants $A$ and $B$ (without loss of generality, suppose $A \neq 0$ ), such that they are not all zero and (see [12])

$$
A x^{(1-2 \lambda)(p-1)} y^{2 \lambda-1} f^{p}(x)=B y^{(1-2 \lambda)(q-1)} x^{2 \lambda-1} g^{q}(y) \text { a.e. in }(0, \infty) \times(0, \infty),
$$

i.e., $A x^{p(1-2 \lambda)} f^{p}(x)=B y^{q(1-2 \lambda)} g^{q}(y)$ a.e. in $(0, \infty) \times(0, \infty)$, thus there exist a constant $C$, such that

$$
A x^{p(1-2 \lambda)} f^{p}(x)=B y^{q(2 \lambda-1)} g^{q}(y)=C \quad \text { a.e. in }(0, \infty) \times(0, \infty) .
$$

Hence $x^{p(1-2 \lambda)-1} f^{p}(x)=\frac{C}{A x}$, which contradicts the fact that $0<\int_{0}^{\infty} x^{p(1-2 \lambda)-1} f^{p}(x) d x<\infty$. Hence (3.3) takes the form of strict inequality. So we have (3.1).

To prove the best constant factor, for $0<\varepsilon<1$, setting

$$
\widetilde{f}(x)=\left\{\begin{array}{ll}
x^{2 \lambda-1-\frac{\varepsilon}{p}}, & x \in[1, \infty), \\
0, & x \in[0,1),
\end{array} \quad \widetilde{g}(x)= \begin{cases}x^{2 \lambda-1-\frac{\varepsilon}{q}}, & x \in[1, \infty), \\
0, & x \in[0,1),\end{cases}\right.
$$

then

$$
\int_{0}^{\infty} x^{p(1-2 \lambda)-1} \widetilde{f}^{p}(x) d x=\int_{0}^{\infty} x^{q(1-2 \lambda)-1} \widetilde{g}^{q}(x) d x=\int_{1}^{\infty} x^{-(1+\varepsilon)} \mathrm{d} x=\frac{1}{\varepsilon} .
$$


For $\theta=(\lambda, a, b, c) \in R_{+}^{4}(a, b, c$ is not equal each other $)$, assume that the constant factor $K(\theta)$ in (3.1) is not the best possible. Then there exists a positive number $k$ with $k<K(\theta)$, such that (3.1) is still valid if $K(\theta)$ is substituted by $k$. In particular, by $(2.5)$, we obtain

$$
\begin{aligned}
K(\theta)+o(1) & =\varepsilon \int_{0}^{\infty} \int_{0}^{\infty} \frac{\tilde{f}(x) \widetilde{g}(y) d x d y}{\left(x^{\lambda}+a y^{\lambda}\right)\left(x^{\lambda}+b y^{\lambda}\right)\left(x^{\lambda}+c y^{\lambda}\right)^{2}} \\
& <\varepsilon k\left\{\int_{0}^{\infty} x^{p(1-2 \lambda)-1} \widetilde{f}^{p}(x) d x\right\}^{1 / p}\left\{\int_{0}^{\infty} y^{q(1-2 \lambda)-1} \widetilde{g}^{q}(x) d x\right\}^{1 / q}=k
\end{aligned}
$$

thus $K(\theta) \leq k$ when $\varepsilon \rightarrow 0^{+}$, which contradicts the hypothesis of $k<K(\theta)$. Hence the constant factor $K(\theta)$ in (3.1) is the best possible for all the $\theta$ which satisfied the conditions.

Theorem 3.2. Under the same conditions of Theorem 3.1. we have

$$
\begin{gathered}
\int_{0}^{\infty} y^{2 \lambda p-1}\left(\int_{0}^{\infty} \frac{f(x) d x}{\left(x^{\lambda}+a y^{\lambda}\right)\left(x^{\lambda}+b y^{\lambda}\right)\left(x^{\lambda}+c y^{\lambda}\right)^{2}}\right)^{p} d y \\
<K^{p}(\theta) \int_{0}^{\infty} x^{p(1-2 \lambda)-1} f^{p}(x) d x
\end{gathered}
$$

where the constant factor $K^{p}(\theta)$ is the best possible. And inequality (3.1) is equivalent to (3.5). In particular, taking $\lambda=1$, we have

$$
\int_{0}^{\infty} y^{2 p-1}\left(\int_{0}^{\infty} \frac{f(x) d x}{(x+a y)(x+b y)(x+c y)^{2}}\right)^{p} d y<K^{p}(1, a, b, c) \int_{0}^{\infty} \frac{1}{x^{p+1}} f^{p}(x) d x,
$$

and inequality (3.6) is equivalent to (3.2).

Proof. For $x \in(0, \infty), n \in N$, setting a bounded measurable function $[f(x)]_{n}$ as

$$
[f(x)]_{n}= \begin{cases}\frac{1}{n}, & f(x)<\frac{1}{n} \\ f(x), & \frac{1}{n} \leqslant f(x) \leqslant n \\ n, & f(x)>n\end{cases}
$$

Setting

$$
\begin{aligned}
g_{n}(y) & :=y^{2 \lambda p-1}\left(\int_{\frac{1}{n}}^{n} \frac{[f(x)]_{n}}{\left(x^{\lambda}+a y^{\lambda}\right)\left(x^{\lambda}+b y^{\lambda}\right)\left(x^{\lambda}+c y^{\lambda}\right)^{2}} d x\right)^{p-1}\left(y \in\left(\frac{1}{n}, n\right)\right) \\
g(y) & :=y^{2 \lambda p-1}\left(\int_{0}^{\infty} \frac{f(x)}{\left(x^{\lambda}+a y^{\lambda}\right)\left(x^{\lambda}+b y^{\lambda}\right)\left(x^{\lambda}+c y^{\lambda}\right)^{2}} d x\right)^{p-1}(y \in(0, \infty)) .
\end{aligned}
$$

Then, there exists $n_{0} \in N$, for $n \geqslant n_{0}, \int_{\frac{1}{n}}^{n} x^{p(1-2 \lambda)-1}[f(x)]_{n}^{p} d x>0,0<$ 
$\int_{\frac{1}{n}}^{n} y^{q(1-2 \lambda)-1} g_{n}^{q}(y) d y<\infty$, and

$$
\begin{aligned}
0 & <\int_{\frac{1}{n}}^{n} y^{q(1-2 \lambda)-1} g_{n}^{q}(y) d y \\
& =\int_{\frac{1}{n}}^{n} y^{2 \lambda p-1}\left(\int_{\frac{1}{n}}^{n} \frac{[f(x)]_{n} d x}{\left(x^{\lambda}+a y^{\lambda}\right)\left(x^{\lambda}+b y^{\lambda}\right)\left(x^{\lambda}+c y^{\lambda}\right)^{2}}\right)^{p} d y \\
& =\int_{\frac{1}{n}}^{n} \int_{\frac{1}{n}}^{n} \frac{[f(x)]_{n} g_{n}(y)}{\left(x^{\lambda}+a y^{\lambda}\right)\left(x^{\lambda}+b y^{\lambda}\right)\left(x^{\lambda}+c y^{\lambda}\right)^{2}} d x d y ; \\
0 & <\int_{0}^{\infty} y^{q(1-2 \lambda)-1} g^{q}(y) d y \\
& =\int_{0}^{\infty} y^{2 \lambda p-1}\left(\int_{0}^{\infty} \frac{f(x) d x}{\left(x^{\lambda}+a y^{\lambda}\right)\left(x^{\lambda}+b y^{\lambda}\right)\left(x^{\lambda}+c y^{\lambda}\right)^{2}}\right)^{p} d y \\
& =\int_{0}^{\infty} \int_{0}^{\infty} \frac{f(x) g(y)}{\left(x^{\lambda}+a y^{\lambda}\right)\left(x^{\lambda}+b y^{\lambda}\right)\left(x^{\lambda}+c y^{\lambda}\right)^{2}} d x d y .
\end{aligned}
$$

By (3.10) and (3.1), we obtain

$$
\begin{aligned}
& \int_{\frac{1}{n}}^{n} y^{q(1-2 \lambda)-1} g_{n}^{q}(y) d y \\
& (3.12)<K(\theta)\left\{\int_{\frac{1}{n}}^{n} x^{p(1-2 \lambda)-1}[f(x)]_{n}^{p} d x\right\}^{1 / p}\left\{\int_{\frac{1}{n}}^{n} y^{q(1-2 \lambda)-1} g_{n}^{q}(y) d y\right\}^{1 / q}<\infty .
\end{aligned}
$$

Hence

$$
0<\left\{\int_{\frac{1}{n}}^{n} y^{q(1-2 \lambda)-1} g_{n}^{q}(y) d y\right\}^{1 / p}<K(\theta)\left\{\int_{\frac{1}{n}}^{n} x^{p(1-2 \lambda)-1}[f(x)]_{n}^{p} d x\right\}^{1 / p}<\infty .
$$

Letting $n \rightarrow \infty$, we have $0<\int_{0}^{\infty} y^{q(1-2 \lambda)-1} g^{q}(y) d y<\infty$.

Similar to the above deduction, applying (3.11) and (3.1) with $f(x), g(y)$, we have

$$
\left\{\int_{0}^{\infty} y^{q(1-2 \lambda)-1} g^{q}(y) d y\right\}^{1 / p}<K(\theta)\left\{\int_{0}^{\infty} x^{p(1-2 \lambda)-1} f^{p}(x) d x\right\}^{1 / p}<\infty,
$$

and we get (3.5) by (3.11) and (3.13).

For $p>1$, by Hölder's inequality, we find

$$
\begin{aligned}
\int_{0}^{\infty} & \int_{0}^{\infty} \frac{f(x) g(y)}{\left(x^{\lambda}+a y^{\lambda}\right)\left(x^{\lambda}+b y^{\lambda}\right)\left(x^{\lambda}+c y^{\lambda}\right)^{2}} d x d y \\
= & \int_{0}^{\infty}\left(y^{2 \lambda-\frac{1}{p}} \int_{0}^{\infty} \frac{f(x) d x}{\left(x^{\lambda}+a y^{\lambda}\right)\left(x^{\lambda}+b y^{\lambda}\right)\left(x^{\lambda}+c y^{\lambda}\right)^{2}}\right)\left(y^{\frac{1}{p}-2 \lambda} g(y)\right) d y \\
\leq & \left\{\int_{0}^{\infty} y^{2 \lambda p-1}\left(\int_{0}^{\infty} \frac{f(x) d x}{\left(x^{\lambda}+a y^{\lambda}\right)\left(x^{\lambda}+b y^{\lambda}\right)\left(x^{\lambda}+c y^{\lambda}\right)^{2}}\right)^{p} d y\right\}^{\frac{1}{p}} \\
& \times\left\{\int_{0}^{\infty} y^{q(1-2 \lambda)-1} g^{q}(y) d y\right\}^{\frac{1}{q}}
\end{aligned}
$$


If (3.5) is valid, then (3.1) is correct by (3.14). Thus (3.1) is equivalent to (3.5).

Assuming that the constant factor $K^{p}(\theta)$ in (3.5) is not the best possible, by (3.14), we may get a contradiction that the constant factor $K(\theta)$ in (3.1) is not the best possible. This completes the proof.

\section{The reverse forms}

Theorem 4.1. If $p<0$ or $0<p<1, \frac{1}{p}+\frac{1}{q}=1, \theta=(\lambda, a, b, c) \in R_{+}^{4}, a, b, c$ is not equal each other, $K(\theta)$ is taken as the definition of (2.3), $f(x), g(x) \geq 0$ such that $0<\int_{0}^{\infty} x^{p(1-2 \lambda)-1} f^{p}(x) d x<\infty$ and $0<\int_{0}^{\infty} y^{q(1-2 \lambda)-1} g^{q}(x) d x<\infty$, then

$$
\begin{aligned}
& \int_{0}^{\infty} \int_{0}^{\infty} \frac{f(x) g(y)}{\left(x^{\lambda}+a y^{\lambda}\right)\left(x^{\lambda}+b y^{\lambda}\right)\left(x^{\lambda}+c y^{\lambda}\right)^{2}} d x d y \\
& \quad>K(\theta)\left\{\int_{0}^{\infty} x^{p(1-2 \lambda)-1} f^{p}(x) d x\right\}^{1 / p}\left\{\int_{0}^{\infty} x^{q(1-2 \lambda)-1} g^{q}(x) d x\right\}^{1 / q}
\end{aligned}
$$

where the constant factor $K(\theta)$ is the best possible. In particular, taking $\lambda=1$, we have

$$
\begin{aligned}
\int_{0}^{\infty} \int_{0}^{\infty} \frac{f(x) g(y)}{(x+a y)(x+b y)(x+c y)^{2}} d x d y \\
\quad>K(1, a, b, c)\left\{\int_{0}^{\infty} \frac{1}{x^{p+1}} f^{p}(x) d x\right\}^{1 / p}\left\{\int_{0}^{\infty} \frac{1}{x^{q+1}} g^{q}(x) d x\right\}^{1 / q} .
\end{aligned}
$$

Proof. By $p<0$ or $0<p<1$, similar to the formulation of (3.3), applying the reverse Hölder's inequality with weight (see [12]), we have the reverse strict inequality as follows

$$
\begin{aligned}
\int_{0}^{\infty} & \int_{0}^{\infty} \frac{f(x) g(y)}{\left(x^{\lambda}+a y^{\lambda}\right)\left(x^{\lambda}+b y^{\lambda}\right)\left(x^{\lambda}+c y^{\lambda}\right)^{2}} d x d y \\
& >\left\{\int_{0}^{\infty} \omega_{1}(x, \theta) x^{p(1-2 \lambda)-1} f^{p}(x) d x\right\}^{1 / p}\left\{\int_{0}^{\infty} \omega_{2}(y, \theta) y^{q(1-2 \lambda)-1} g^{q}(y) d y\right\}^{1 / q} \\
(4.3) & =K(\theta)\left\{\int_{0}^{\infty} x^{p(1-2 \lambda)-1} f^{p}(x) d x\right\}^{1 / p}\left\{\int_{0}^{\infty} x^{q(1-2 \lambda)-1} g^{q}(x) d x\right\}^{1 / q} .
\end{aligned}
$$

Thus (4.1) is valid. Suppose there exist a positive number $K_{0} \geq K(\theta)$, such that (4.1) is still valid that $K(\theta)$ is instead of $K_{0}$. In particular, (4.1) is valid for the function $\widetilde{f}(x), \widetilde{g}(y)$ which is defined by (3.4), combining (2.5), we have

$$
\begin{aligned}
K(\theta)+o(1) & =\varepsilon \int_{0}^{\infty} \int_{0}^{\infty} \frac{\widetilde{f}(x) \widetilde{g}(y) d x d y}{\left(x^{\lambda}+a y^{\lambda}\right)\left(x^{\lambda}+b y^{\lambda}\right)\left(x^{\lambda}+c y^{\lambda}\right)^{2}} \\
& >\varepsilon K_{0}\left\{\int_{0}^{\infty} x^{p(1-2 \lambda)-1} \widetilde{f}^{p}(x) d x\right\}^{1 / p}\left\{\int_{0}^{\infty} x^{q(1-2 \lambda)-1} \widetilde{g}^{q}(x) d x\right\}^{1 / q}=K_{0},
\end{aligned}
$$


thus $K(\theta) \geq K_{0}$, when $\varepsilon \rightarrow 0^{+}$. Hence $K_{0}=K(\theta)$ and the constant factor $K(\theta)$ in (4.1) is the best possible.

Theorem 4.2. Under the same conditions of Theorem 4.1. we have

(i) for $p<0$,

$$
\begin{gathered}
\int_{0}^{\infty} y^{2 \lambda p-1}\left(\int_{0}^{\infty} \frac{f(x) d x}{\left(x^{\lambda}+a y^{\lambda}\right)\left(x^{\lambda}+b y^{\lambda}\right)\left(x^{\lambda}+c y^{\lambda}\right)^{2}}\right)^{p} d y \\
<K^{p}(\theta) \int_{0}^{\infty} x^{p(1-2 \lambda)-1} f^{p}(x) d x,
\end{gathered}
$$

where the constant factor $K^{p}(\theta)$ is the best possible, and (4.1) is equivalent to (4.4);

(ii) for $0<p<1$,

$$
\begin{gathered}
\int_{0}^{\infty} y^{2 \lambda p-1}\left(\int_{0}^{\infty} \frac{f(x) d x}{\left(x^{\lambda}+a y^{\lambda}\right)\left(x^{\lambda}+b y^{\lambda}\right)\left(x^{\lambda}+c y^{\lambda}\right)^{2}}\right)^{p} d y \\
>K^{p}(\theta) \int_{0}^{\infty} x^{p(1-2 \lambda)-1} f^{p}(x) d x,
\end{gathered}
$$

where the constant factor $K^{p}(\theta)$ is the best possible, and (4.1) is equivalent to (4.5).

Proof. For $p<0$ or $0<p<1$, by the reverse Hölder's inequality, we get

$$
\begin{aligned}
\int_{0}^{\infty} & \int_{0}^{\infty} \frac{f(x) g(y)}{\left(x^{\lambda}+a y^{\lambda}\right)\left(x^{\lambda}+b y^{\lambda}\right)\left(x^{\lambda}+c y^{\lambda}\right)^{2}} d x d y \\
= & \int_{0}^{\infty}\left(y^{2 \lambda-\frac{1}{p}} \int_{0}^{\infty} \frac{f(x) d x}{\left(x^{\lambda}+a y^{\lambda}\right)\left(x^{\lambda}+b y^{\lambda}\right)\left(x^{\lambda}+c y^{\lambda}\right)^{2}}\right)\left(y^{\frac{1}{p}-2 \lambda} g(y)\right) d y \\
\geq & \left\{\int_{0}^{\infty} y^{2 \lambda p-1}\left(\int_{0}^{\infty} \frac{f(x) d x}{\left(x^{\lambda}+a y^{\lambda}\right)\left(x^{\lambda}+b y^{\lambda}\right)\left(x^{\lambda}+c y^{\lambda}\right)^{2}} d y\right\}^{\frac{1}{p}}\right. \\
& \times\left\{\int_{0}^{\infty} y^{q(1-2 \lambda)-1} g^{q}(y) d y\right\}^{\frac{1}{q}}
\end{aligned}
$$

Setting $[f(x)]_{n}$ and $g_{n}(y)$ as the definition of (3.7) and (3.8), then, there exists $n_{0} \in$ $N$, for $n \geqslant n_{0}, \int_{\frac{1}{n}}^{n} x^{p(1-2 \lambda)-1}[f(x)]_{n}^{p} d x>0$ and $0<\int_{\frac{1}{n}}^{n} y^{q(1-2 \lambda)-1} g_{n}^{q}(y) d y<\infty$.

(i) For $p<0$, by (3.10) and (4.1), we obtain

$$
\begin{aligned}
\infty & >\int_{\frac{1}{n}}^{n} y^{q(1-2 \lambda)-1} g_{n}^{q}(y) d y \\
& >K(\theta)\left\{\int_{\frac{1}{n}}^{n} x^{p(1-2 \lambda)-1}[f(x)]_{n}^{p} d x\right\}^{1 / p}\left\{\int_{\frac{1}{n}}^{n} y^{q(1-2 \lambda)-1} g_{n}^{q}(y) d y\right\}^{1 / q}>0 .
\end{aligned}
$$

Hence

$$
\infty>\left\{\int_{\frac{1}{n}}^{n} y^{q(1-2 \lambda)-1} g_{n}^{q}(y) d y\right\}^{1 / p}>K(\theta)\left\{\int_{\frac{1}{n}}^{n} x^{p(1-2 \lambda)-1}[f(x)]_{n}^{p} d x\right\}^{1 / p}>0,
$$




$$
\int_{\frac{1}{n}}^{n} y^{q(1-2 \lambda)-1} g_{n}^{q}(y) d y<K^{p}(\theta) \int_{\frac{1}{n}}^{n} x^{p(1-2 \lambda)-1}[f(x)]_{n}^{p} d x<\infty .
$$

Letting $n \rightarrow \infty$, we have $0<\int_{0}^{\infty} y^{q(1-2 \lambda)-1} g^{q}(y) d y<\infty$. By the same deduction, applying (3.11) and (4.1) with $f(x), g(y)$, we have

$$
\int_{0}^{\infty} y^{q(1-2 \lambda)-1} g^{q}(y) d y<K^{p}(\theta) \int_{0}^{\infty} x^{p(1-2 \lambda)-1} f^{p}(x) d x
$$

combining (3.11), we get (4.4) for $p<0$.

Suppose that (4.4) is valid. By (4.6), (4.1) is correct for $p<0$. Thus (4.1) is equivalent to (4.4).

If the constant factor $K^{p}(\theta)$ in (4.4) is not the best possible, then by (4.6) $(p<0)$, we may get a contradiction that the constant factor $k(\theta)$ in $(4.1)$ is not the best possible.

(ii) For $0<p<1$, suppose that (4.5) is valid. By (4.6), (4.1) is valid too.

Assume that (4.1) is valid. If $\int_{0}^{\infty} y^{q(1-2 \lambda)-1} g^{q}(y) d y=\infty$, then by (3.11) and $\int_{0}^{\infty} x^{p(1-2 \lambda)-1} f^{p}(x) d x<\infty$, we get $(4.5)$; if $0<\int_{0}^{\infty} y^{q(1-2 \lambda)-1} g^{q}(y) d y<\infty$, then by (3.11) and (4.1), we have

(4.9)

$\int_{0}^{\infty} y^{q(1-2 \lambda)-1} g^{q}(y) d y>K(\theta)\left\{\int_{0}^{\infty} x^{p(1-2 \lambda)-1} f^{p}(x) d x\right\}^{\frac{1}{p}}\left\{\int_{0}^{\infty} y^{q(1-2 \lambda)-1} g^{q}(y) d y\right\}^{\frac{1}{q}}$.

Thus

$$
\int_{0}^{\infty} y^{q(1-2 \lambda)-1} g^{q}(y) d y>K^{p}(\theta) \int_{0}^{\infty} x^{p(1-2 \lambda)-1} f^{p}(x) d x .
$$

By (3.11), the inequality above turns into (4.5). Hence (4.1) is equivalent to (4.5).

If the constant factor $K^{p}(\theta)$ in (4.5) is not the best possible, then by (4.6) $(0<p<1)$, we may get a contradiction that the constant factor $K(\theta)$ in $(4.1)$ is not the best possible.

Remarks. (i) Setting $h(x)=\frac{x \ln (c / x)}{(x-c)^{2}}$, we have

$$
\begin{aligned}
K(\lambda, a, a, c) & :=\lim _{b \rightarrow a} K(\theta)=\lim _{b \rightarrow a} \frac{1}{\lambda}\left[\frac{1}{(a-c)(c-b)}+\frac{h(b)-h(a)}{b-a}\right] \\
& =\frac{1}{\lambda}\left[h^{\prime}(a)-\frac{1}{(a-c)^{2}}\right]=\frac{(a+c)}{\lambda(a-c)^{2}}\left[\frac{\ln (a / c)}{a-c}-\frac{2}{(a+c)}\right](c \neq a) \\
K(\lambda, a, b, a) & :=\lim _{c \rightarrow a} K(\lambda, a, b, c)=\frac{b}{\lambda(b-a)^{2}}\left[\frac{b+a}{2 a b}-\frac{\ln (b / a)}{b-a}\right](b \neq a) ; \\
K(\lambda, a, a, a) & :=\lim _{b \rightarrow a} K(\lambda, a, b, a)=\frac{1}{6 \lambda a^{2}} .
\end{aligned}
$$


Taking $\lambda=1$, we obtain the following inequalities by (3.1)

$$
\begin{aligned}
& \int_{0}^{\infty} \int_{0}^{\infty} \frac{f(x) g(y)}{(x+a y)^{2}(x+c y)^{2}} d x d y \\
&<K(1, a, a, c)\left\{\int_{0}^{\infty} \frac{1}{x^{p+1}} f^{p}(x) d x\right\}^{1 / p}\left\{\int_{0}^{\infty} \frac{1}{x^{q+1}} g^{q}(x) d x\right\}^{1 / q} \\
& \int_{0}^{\infty} \int_{0}^{\infty} \frac{f(x) g(y)}{(x+a y)^{3}(x+b y)} d x d y \\
& \quad<K(1, a, b, a)\left\{\int_{0}^{\infty} \frac{1}{x^{p+1}} f^{p}(x) d x\right\}^{1 / p}\left\{\int_{0}^{\infty} \frac{1}{x^{q+1}} g^{q}(x) d x\right\}^{1 / q}
\end{aligned}
$$

$$
\int_{0}^{\infty} \int_{0}^{\infty} \frac{f(x) g(y)}{(x+a y)^{4}} d x d y<\frac{1}{6 a^{2}}\left\{\int_{0}^{\infty} \frac{1}{x^{p+1}} f^{p}(x) d x\right\}^{1 / p}\left\{\int_{0}^{\infty} \frac{1}{x^{q+1}} g^{q}(x) d x\right\}^{1 / q} .
$$

(ii) The kernel of (3.2) is the homogeneous of -4-degree, it is a new Hilberttype integral inequality with the best constant factor, and (3.1) can be taken as the best extension of (3.2). Similarly, (3.1) can be taken as the best extension of (4.10)-(4.12).

(iii) Taking the parameter $p=q=2, a=1$, we get (1.5) by (4.12), thus (3.1) is the best extension of (1.5) with multi-parameter.

Acknowledgment. This work was supported by the Emphases Natural Science Foundation of Guangdong Institutions of Higher Learning, College and University (No. 05Z026). The authors would like to thank the anonymous referee for his or her suggestions and corrections.

\section{References}

[1] G. H. Hardy, J. E. Littlewood, and G. Pólya, Inequalities, Cambridge Univ. Press, Cambridge, UK, 2nd edition, 1952.

[2] D. S. Mitrinović, J. E. Pečarić and A. M. Fink, Inequalities involving functions and their integrals and derivatives, Kluwer Acad. Publ., Boston, 1991.

[3] B. Yang, On Hilbert's integral inequality, J. Math. Anal. Appl., 220(1998), 778-785.

[4] B. Yang, A Note on Hilbert's Integral Inequalities, Chin Quar. J. Math., 13(4)(1998), 83-86.

[5] B. Yang, L. Debnath, On the Extended Hardy-Hilbert's Inequality, J. Math. Anal. Appl., 272(1)(2002), 187-199.

[6] B. Yang, I. Brnetić, M. Krnić, J. Pečarić, Generalization of Hilbert and Hardy-Hilbert integral inequalities, Math. Ineq. Appl., 8(2)(2005), 259-272. 
[7] Y. Hong, On Hardy-Hilbert integral inequalities with some parameters, J. Ineq. in Pure and Applied Math., 6(4)(2005), Art. 92, 1-10.

[8] B. Yang, On Hilbert's Inequality with Some Parameters, Acta Mathematica Sinica, 49(5)(2006), 1121-1126.

[9] B. Yang, On a Reverse of Hardy-Hilbert's Integral Inequality, Pure and Applied Mathematics, 22(3)(2006), 312-317.

[10] B. Yang, A Bilinear Inequality with the Kernal of -2-order Homogeneous, Journal of Xiamen University(Natural Science), 45(6)(2006), 752-755.

[11] Z. Xie, Z. Zheng, A Hilbert-type integral inequality whose kernel is a homogeneous form of degree -3, J. Math. Anal. Appl., 339(1)(2007), 324-331.

[12] J. Kuang, Applied inequalities, Shandong Science and Technology Press, Jinan, China, 2004 